\title{
ON CLOSED CONVEX SURFACES
}

\author{
V. G. GROVE
}

1. Introduction. The purpose of this paper is to prove the following theorem. Let $S$ and $\bar{S}$ be two closed orientable convex surfaces of class $C^{\prime \prime \prime}$ imbedded in an euclidian space $E^{3}$ of three dimensions, and possessing no parabolic points. Let $h$ be a differentiable homeomorphism of $S$ into $\bar{S}$ such that (a) II $=\overline{\mathrm{I}} \overline{\mathrm{I}}$, II and $\overline{\mathrm{I}} \overline{\mathrm{I}}$ being the second fundamental forms of $S$ and $\bar{S}$ respectively, (b) such that the Gaussian curvatures $K$ and $\bar{K}$ of $S$ and $\bar{S}$ are equal at corresponding points $X$ and $\bar{X}$, and (c) such that the orientations of $S$ and $\bar{S}$ are preserved. Then $h$ is a rigid motion.

Incidental to the proof of the theorem, we present a simple proof of Liebmann's theorem on the rigidity of the sphere. In seeking an integral formula furnishing a proof of the theorem an integral formula was found which gave a simple proof of the fact that such a surface $S$, described in the theorem and for which the ratio of the mean to the Gaussian curvature is a constant, is a sphere. Such a surface is of course a "special" Weingarten surface. Chern has proved [3] that all convex special $W$-surfaces are spheres. Hence our statement is but a special case of Chern's theorem.

Since $S$ and $\bar{S}$ are orientable, we may assume that their second fundamental forms are positive definite.

2. Exterior forms on $S$. Let $0-I_{1}, I_{2}, I_{3}$ be a fixed orthogonal frame in $E^{3}$. Let $\left(x^{1}, x^{2}, x^{3}\right)$ be the coordinates of a point $X$ in $E^{3}$ with respect to this orthogonal frame. The vector equation of the surface $S$ has the form

$$
X=X\left(u^{1}, u^{2}\right),
$$

wherein the components $\left(x^{1}, x^{2}, x^{3}\right)$ of the position vector $X$ are of class $C^{\prime \prime \prime}$ in a simply connected domain $D$ of a parameter plane. Moreover the vector $X_{1} \times X_{2}$ wherein $X_{\alpha}=\left(\partial X / \partial u^{\alpha}\right)$, is not a null vector for any point of $D$.

We shall use the usual summation convention: repeated indices indicating summation over the range of the indices. We shall let the roman letters have the range $1,2,3$ and the greek letters the range $1,2$.

Presented to the Society December 1, 1956; received by the editors September 24, 1956. 
Let $E_{3}$ be the unit normal vector of $S$. The first and second fundamental forms of $S$ are given by

$$
\begin{aligned}
\mathrm{I} & =(d X)^{2}=X_{\rho} \cdot X_{\sigma} d u^{\rho} d u^{\sigma}=g_{\rho \sigma} d u^{\rho} d u^{\sigma}, \\
\mathrm{II} & =-d E_{3} \cdot d X=d_{\rho \sigma} d u^{\rho} d u^{\sigma} .
\end{aligned}
$$

Let $X-E_{1}, E_{2}, E_{3}$ be a frame, to be called a conjugate frame, such that $\left(E_{1}, E_{2}, E_{3}\right)>0$, and such that if

$$
E_{1}=U^{\rho} X_{\rho}, \quad E_{2}=V^{\rho} X_{\rho},
$$

then

$$
d_{\rho \sigma} U^{\rho} U^{\sigma}=1, \quad d_{\rho \sigma} U^{\rho} V^{\sigma}=0, \quad d_{\rho \sigma} V^{\rho} V^{\sigma}=1,
$$

wherein $U^{\alpha}, V^{\alpha}$ are functions of $u^{1} u^{2}$ Class $C^{\prime \prime}$. Conditions (2.4) imply that the tangent vectors $E_{1}, E_{2}$ form an orthonormal frame with respect to the metric II. They are conjugate vectors in the sense of Dupin.

Let

$$
E_{1}^{2}=a, \quad E_{1} \cdot E_{2}=b, \quad E_{2}^{2}=c .
$$

Let $\omega^{1}, \omega^{2}$ be two forms on $S$ defined by

$$
\omega^{1}=U_{\rho} d u^{\rho}, \quad \omega^{2}=V_{\rho} d u^{\rho}, \quad U_{\alpha}=d_{\alpha \rho} U^{\rho}, \quad V_{\alpha}=d_{\alpha \rho} V^{\rho} .
$$

From (2.3) and (2.6) we find that

$$
X_{\alpha}=U_{\alpha} E_{1}+V_{\alpha} E_{2}, \quad d u^{\alpha}=U^{\alpha} \omega^{1}+V^{\alpha} \omega^{1} .
$$

Hence

(2.8) $d X=X_{\rho} d u^{\rho}=\left(U_{\rho} E_{1}+V_{\rho} E_{2}\right)\left(U^{\rho} \omega^{1}+V^{\rho} \omega^{2}\right)=\omega^{i} E_{i}, \omega^{3}=0$.

We also write

$$
d E_{i}=\omega_{i}^{i} E_{j}, \quad \omega_{3}^{3}=0 .
$$

Taking exterior differentials of (2.8) and (2.9), and using (2.8) and (2.9) we find that

$$
d \omega^{i}=\omega^{j} \wedge \omega_{j}^{i}, \quad d \omega_{j}^{i}=\omega_{j}^{k} \wedge \omega_{k}^{i}
$$

Equations (2.10) are of course the conditions of compatibility of (2.8) and (2.9).

Using (2.4) and (2.5) we find that the first and second fundamental forms of $S$ are

$$
\mathrm{I}=a\left(\omega^{1}\right)^{2}+2 b \omega^{1} \omega^{2}+c\left(\omega^{2}\right)^{2}, \quad \mathrm{II}=\left(\omega^{1}\right)^{2}+\left(\omega^{2}\right)^{2} .
$$


It follows from (2.11) that the mean $H$ of the principal normal curvatures and the Gaussian curvature $K$ of $S$ are given by

$$
2 H=(a+c) K, \quad\left(a c-b^{2}\right) K=1 .
$$

Since $\omega^{3}=0$, we find from the first of $(2.10)$ that

$$
\omega^{1} \wedge \omega_{1}^{3}+\omega^{2} \wedge \omega_{2}^{3}=0 .
$$

Hence $\omega_{1}^{3}$ and $\omega_{2}^{3}$ must have the form

$$
\dot{\omega}_{1}^{3}=p \omega^{1}+q \omega^{2}, \quad \omega_{2}^{3}=q \omega^{1}+r \omega^{2} .
$$

Since $E_{1} \cdot E_{3}=0, E_{2} \cdot E_{3}=0$, it follows from (2.9) that

$$
\underset{\omega_{3}}{i} E_{1} \cdot E_{i}+\underset{\omega_{1}^{i}}{E_{i}} \cdot E_{3}=0, \quad \omega_{3}^{i} E_{2} \cdot E_{i}+\omega_{2}^{i} E_{i} \cdot E_{3}=0 .
$$

Hence

$$
{\stackrel{3}{\omega_{1}}}^{3}=-\left(a \omega_{3}^{1}+b \omega_{3}^{2}\right), \quad \stackrel{\omega}{3}_{2}^{3}=-\left(b \omega_{3}^{1}+c \omega_{3}^{2}\right) .
$$

From (2.2) the second fundamental form of $S$ is given by

$$
\mathrm{II}=-d E_{3} \cdot d X=-\left[\left(a \omega_{3}^{1}+b \omega_{3}^{2}\right) \omega^{1}+\left(b \omega_{3}^{1}+c \omega_{3}^{2}\right) \omega^{2}\right]=\omega_{1}^{3} \omega^{1}+\omega_{2}^{3} \omega^{2} .
$$

But from (2.13) and the second of (2.11) we find that $p=r=1, q=0$. Therefore (2.13) and (2.14) assume the form

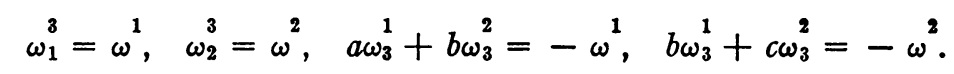

From the last two of (2.15) we find that

$$
\omega_{3}^{1}=K\left(-c \omega^{1}+b \omega^{2}\right), \quad \omega_{3}^{2}=K\left(b \omega^{1}-a \omega^{2}\right) .
$$

Taking exterior differentials of the first two of (2.15) we find that

(2.17) $2 \omega_{1}^{1} \wedge \omega^{1}=\omega^{2} \wedge\left(\omega_{2}^{1}+\omega_{1}^{2}\right), 2 \omega_{2}^{2} \wedge \omega^{2}=\omega^{1} \wedge\left(\omega_{2}^{1}+\omega_{1}^{2}\right)$.

It follows from (2.17) that $\omega_{1}^{1}, \omega_{2}^{2}, \omega_{2}^{1}+\omega_{1}^{2}$ have the following form

$$
\begin{aligned}
\omega_{1}^{1} & =A \omega^{1}+B \omega^{2}, \\
\omega_{2}^{2} & =A^{\prime} \omega^{1}+B^{\prime} \omega^{2}, \\
\omega_{2}^{1}+\omega_{1}^{2} & =2\left(B \omega^{1}+A^{\prime} \omega^{2}\right) .
\end{aligned}
$$

From (2.5) and (2.9) we find that 


$$
\begin{gathered}
d a=2\left(a \omega_{1}^{1}+b \omega_{1}^{2}\right), \quad d c=2\left(b \omega_{2}^{1}+c \omega_{2}^{2}\right), \\
d b=b\left(\omega_{1}^{1}+\omega_{2}^{2}\right)+a \omega_{2}^{1}+c \omega_{1}^{2} .
\end{gathered}
$$

Hence

$$
d\left(a c-b^{2}\right)=2\left(\omega_{1}^{1}+\omega_{2}^{2}\right)\left(a c-b^{2}\right) .
$$

Therefore

$$
d K=-2\left(\omega_{1}^{1}+\omega_{2}^{2}\right) K .
$$

3. An associated Riemannian space $\Re$. Consider a Riemannian space $\Re$ defined over $D$ whose metric is given by

$$
d s^{2}=\mathrm{II}=\left(\omega^{1}\right)^{2}+\left(\omega^{2}\right)^{2} .
$$

We shall call this space the associated Riemannian space.

As is well known for Riemannian spaces [2] there exists an unique form $\psi_{1}^{2}\left(=-\psi_{2}^{1}\right)$ such that

$$
d \omega^{1}=\omega^{2} \wedge \psi_{2}^{1}, \quad d \omega^{2}=\omega^{1} \wedge \psi_{1}^{2} .
$$

That this form is unique follows from assuming there are two forms $\psi_{2}^{1}, \psi_{2}^{1}$ satisfying (3.2). That is, not only does (3.2) hold but also

$$
d \omega^{1}=\omega^{2} \wedge \bar{\psi}_{2}^{1}, \quad d \omega^{2}=\omega^{1} \wedge \bar{\psi}_{1}^{2} .
$$

Subtracting (3.2) from the above it follows that

$$
\begin{aligned}
& \omega^{2} \wedge\left(\bar{\psi}_{2}^{1}-\psi_{2}^{1}\right)=0, \\
& \omega^{1} \wedge\left(\bar{\psi}_{1}^{2}-\psi_{1}^{2}\right)=0 .
\end{aligned}
$$

But since $\omega^{1}, \omega^{2}$ are linearly independent, $\psi_{1}^{2}=\psi_{1}^{2}$. From the first of (2.10) with first $i=1$, then $i=2$ and using (2.17) we find readily that

$$
\psi_{1}^{2}=\frac{1}{2}\left(\omega_{1}^{2}-\omega_{2}^{1}\right)
$$

satisfies (3.2) and hence is the desired unique form.

Using (2.10) we find that

$$
d \psi_{1}^{2}=\frac{1}{2}\left[\left(\omega_{1}^{1}-\omega_{2}^{2}\right) \wedge\left(\omega_{2}^{1}+\omega_{1}^{2}\right)+\omega^{1} \wedge \omega_{3}^{2}-\omega^{2} \wedge \omega_{3}^{1}\right] .
$$

Hence from (2.12), (2.16) and (2.18) we find that 


$$
d \psi_{1}^{2}=-(G+H) \omega^{1} \wedge \omega^{2},
$$

wherein $H$ is the mean curvature of $S$ and

$$
G=A^{\prime}\left(A^{\prime}-A\right)+B\left(B-B^{\prime}\right) \text {. }
$$

It follows that

$$
\Re=G+H
$$

is the Gaussian curvature of the associated Riemannian space. We shall call this curvature the associated curvature of $S$. The associated curvatures of all surfaces having the same second fundamental forms are of course the same.

4. Integral formulas on $S$. Before developing the formulas we shall use to prove the theorem, it will be necessary to consider the effect on the functions $a, b, c$ and the forms $\omega^{i}, \omega_{j}^{i}$ by a change of conjugate frame.

Let $F^{\prime}=X-E_{1}^{\prime}, E_{2}^{\prime}, E_{3}^{\prime}, E_{3}^{\prime}=E_{3}$ be a second conjugate frame. Letting

$$
E_{1}^{\prime}=U^{\prime} \rho X_{\rho}, \quad E_{2}^{\prime}=V^{\prime} \rho X_{\rho},
$$

and noting that $E_{1}^{\prime}, E_{2}^{\prime}$ have the same orientation as $E_{1}, E_{2}$, and are also orthonormal with respect to II (cf. 2.4) we may write

$$
E_{1}^{\prime}=E_{1} \cos \theta+E_{2} \sin \theta, \quad E_{2}^{\prime}=-E_{1} \sin \theta+E_{2} \cos \theta,
$$

$\theta$ being a function of $u^{1}, u^{2}$ of class $C^{\prime}$.

The functions $a^{\prime}, b^{\prime}, c^{\prime}$ corresponding to $a, b, c$ are readily found to be given by

$$
\begin{aligned}
a^{\prime} & =a \cos ^{2} \theta+b \sin 2 \theta+c \sin ^{2} \theta \\
2 b^{\prime} & =(c-a) \sin 2 \theta+2 b \cos 2 \theta \\
c^{\prime} & =a \sin ^{2} \theta-b \sin 2 \theta+c \cos ^{2} \theta
\end{aligned}
$$

Since

$$
U^{\prime \alpha}=U^{\alpha} \cos \theta+V^{\alpha} \sin \theta, \quad V^{\prime \alpha}=-U^{\alpha} \sin \theta+V^{\alpha} \cos \theta
$$

it follows that, if $\phi^{i}, \phi_{j}^{i}$ are the forms corresponding to $\omega^{i}, \omega_{j}^{i}$,

$$
\phi^{1}=\omega^{1} \cos \theta+\omega^{2} \sin \theta, \quad \phi^{2}=-\omega^{1} \sin \theta+\omega^{2} \cos \theta .
$$

Moreover

$$
\phi^{1} \wedge \phi^{2}+\omega^{1} \wedge \omega^{2}
$$

Direct computation from (4.1) gives 


$$
\begin{aligned}
& \phi_{1}^{1}=\omega_{1}^{1} \cos ^{2} \theta+\frac{1}{2}\left(\omega_{2}^{1}+\omega_{1}^{2}\right) \sin 2 \theta+\omega_{2}^{2} \sin ^{2} \theta, \\
& \phi_{2}^{2}=\omega_{1}^{1} \sin ^{2} \theta-\frac{1}{2}\left(\omega_{2}^{1}+\omega_{1}^{2}\right) \sin 2 \theta+\omega_{2}^{2} \cos ^{2} \theta,
\end{aligned}
$$

$$
\begin{aligned}
& \phi_{1}^{2}=\frac{1}{2}\left(\omega_{2}^{2}-\omega_{1}^{1}\right) \sin 2 \theta+\omega_{1}^{2} \cos ^{2} \theta-\omega_{2}^{1} \sin ^{2} \theta+d \theta, \\
& \phi_{2}^{1}=\frac{1}{2}\left(\omega_{2}^{2}-\omega_{1}^{1}\right) \sin 2 \theta-\omega_{1}^{2} \sin ^{2} \theta+\omega_{2}^{1} \cos ^{2} \theta-d \theta, \\
& \phi_{3}^{1}=\omega_{3}^{1} \cos \theta+\omega_{3}^{2} \sin \theta, \quad \phi_{3}^{2}=-\omega_{3}^{1} \sin \theta+\omega_{3}^{2} \cos \theta .
\end{aligned}
$$

It follows from the third and fourth of (4.5) that $d \psi_{1}^{2}$ is independent of the conjugate frame $F$, a fact which is geometrically evident. that

We find it convenient at this point to note, using (2.4) and (2.6),

$$
\omega^{1} \wedge \omega^{2}=U_{\rho} V_{\sigma} d u^{\rho} \wedge d u^{\sigma}=\left(U_{1} V_{2}-U_{2} V_{1}\right) d u^{1} \wedge d u^{2} .
$$

We note (4) that

$$
U_{1} V_{2}-U_{2} V_{1}=d^{1 / 2},
$$

wherein $d=\operatorname{det}\left(d_{\alpha \beta}\right)$. Hence

$$
\omega^{1} \wedge \omega^{2}=d^{1 / 2} d u^{1} \wedge d u^{2}=K^{1 / 2} g^{1 / 2} d u^{1} \wedge d u^{2}=K^{1 / 2} d A,
$$

$d A$ being "the element of area" of $S$.

Let us now define auxiliary functions $y_{i}$ by the formulas

$$
y_{i}=X \cdot E_{i} .
$$

We find readily that

$$
d y_{1}=a \omega^{1}+b \omega^{2}+\omega_{1}^{j} y_{j}, \quad d y_{2}=b \omega^{1}+c \omega^{2}+\omega_{2}^{j} y_{j} .
$$

We note from (4.2) and (4.5) that the following forms are independent of the frame $F$ :

$$
\begin{aligned}
& \omega_{1}=K^{1 / 2}\left(y_{1} \omega^{2}-y_{2} \omega^{1}\right), \\
& \omega_{2}=K^{1 / 2}\left[y_{1}\left(b \omega^{1}+c \omega^{2}\right)-y_{2}\left(a \omega^{1}+b \omega^{2}\right)\right], \\
& \omega_{3}=K^{1 / 2}\left(z_{1} \omega^{2}-z_{2} \omega^{1}\right), \\
& \omega_{4}=K^{1 / 2}\left[2 b\left(\omega_{1}^{1}-\omega_{2}^{2}\right)+(c-a)\left(\omega_{2}^{1}+\omega_{1}^{2}\right)\right],
\end{aligned}
$$


wherein

$$
z_{1}=(a-c) y_{1}+2 b y_{2}, \quad z_{2}=2 b y_{1}+(c-a) y_{2} .
$$

Stokes' formula applied to a linear form $\omega$ may be written as

$$
\int_{C} \omega=\iint_{R} d \omega
$$

wherein $C$ is the boundary of the simply connected region $R$. Applying this formula to the forms (4.8) in turn, and recalling that $S$ was assumed closed and convex, we find that

$$
\begin{aligned}
& \iint_{S}\left(H+K y_{3}\right) d A=0, \quad \iint_{S}\left(1+H y_{3}\right) d A=0, \\
& \iint_{S} K\left(p+2 f^{\rho} y_{p}\right) d A=0, \quad \iint_{S} K[K p+4(a+c) G] d A=0,
\end{aligned}
$$

wherein

$$
\begin{gathered}
p=a^{2}+c^{2}-2 a c+4 b^{2}, \\
f^{1}=a A+2 b B+c A^{\prime}, \quad f^{2}=a B+2 b A^{\prime}+c B^{\prime} .
\end{gathered}
$$

The first and second of (4.9) are of course the familiar formulas associated with closed surfaces, the first being Minkowski's formula [2].

The first of (4.10) using (2.12) may be written in the form

$$
p=(a-c)^{2}+4 b^{2}=(a+c)^{2}-4\left(a c-b^{2}\right)=4\left(H^{2}-K\right) / K^{2} .
$$

Hence $p \geqq 0$ on $D$, the equality holding only at an umbilical point of $S$.

Using (3.6) we may write the fourth of (4.9) in the form

$$
\iint_{S} H(2 \Re-H) d A=\iint_{S} K d A .
$$

Formula (4.12) relates the mean and associated curvatures of $S$ with the curvatura integra, and hence to the genus of $S$.

Consider now the first of (2.12) written in the form

$$
2 H / K=a+c \text {. }
$$

From (2.18) and (2.19) we find readily that

$$
d(a+c)=2\left(f^{1} \omega^{1}+f^{2} \omega^{2}\right) .
$$

Therefore in case the ratio of the mean to the Gaussian curvature is a constant, the third of (4.9) assumes the form 


$$
\iint_{S} K p d A=0
$$

Hence since $p \geqq 0$, it follows that every point of $S$ is an umbilic, and $S$ is a sphere.

5. The proof of the theorem. The formulas developed in the previous sections have analogous forms for the surface $\bar{S}$ of the theorem. We shall denote the corresponding expressions for $\bar{S}$ by the same but barred letters.

The homeomorphism $h: S \rightarrow \bar{S}$ induces a homeomorphism $h^{*}$ on contravariant tensors at a point $X$ of $S$ and a homeomorphism $h_{*}$ on covariant tensors at a point $X$ of $S$ into contravariant and covariant tensors respectively at $\bar{X}=h X$ on $\bar{S}$.

Let $F=X-E_{1}, E_{2}, E_{3}$ be a given conjugate frame of $S$. We take for the frame of $\bar{S}$, the image of $F$, that is

$$
\bar{F}=h X-h^{*} E_{1}, h^{*} E_{2}, \bar{E}_{3},
$$

$\bar{E}_{3}$ being the unit normal vector of $\bar{S}$. This frame $\bar{F}$ is a conjugate frame on $\bar{S}$ since the form II is preserved under $h$. Using such corresponding frames on $S$ and $\bar{S}$, it follows that

$$
\bar{\omega}^{i}=h_{*} \omega^{i} .
$$

By assumption $K=\bar{K}$, hence from (2.20)

$$
\bar{\omega}_{1}^{1}+\bar{\omega}_{2}^{2}=\omega_{1}^{1}+\omega_{2}^{2} .
$$

From (2.18) and its analogue for $\bar{S}$, it follows that

$$
\bar{A}+\bar{A}^{\prime}=A+A^{\prime}, \quad \bar{B}+\bar{B}^{\prime}=B+B^{\prime} .
$$

Since II = II, from (3.1), (3.3) and (3.5) it follows that

$$
\bar{\omega}_{1}^{2}-\bar{\omega}_{2}^{1}=\omega_{1}^{2}-\omega_{2}^{1} .
$$

Moreover from (4.2) and the analogue of (4.5) for $\bar{S}$, the form

$$
\bar{\omega}=K^{1 / 2}\left[2 b\left(\bar{\omega}_{1}^{1}-\bar{\omega}_{2}^{2}\right)+(c-a)\left(\bar{\omega}_{2}^{1}+\bar{\omega}_{1}^{2}\right)\right]
$$

is independent of the frame $F$, and hence is a meaningful linear form on $S$. Using (5.1), (5.2) and (5.3) we find that

$$
d \bar{\omega}=K\left[C^{\prime} K+4(a+c) G^{\prime}\right] d A,
$$

wherein 


$$
\begin{aligned}
C^{\prime} & =2 \delta+4(H \bar{H}-K) / K^{2}, \\
2 G^{\prime} & =2\left(A^{\prime} \bar{A}^{\prime}+B \bar{B}\right)-\bar{A} A^{\prime}-A \bar{A}^{\prime}-B \bar{B}^{\prime}-\bar{B} B^{\prime}, \\
\delta & =\left|\begin{array}{ll}
a-\bar{a} & b-\bar{b} \\
b-b & c-\bar{c}
\end{array}\right| .
\end{aligned}
$$

Application of Stokes' formula to the form $\bar{\omega}$ over the closed surface $S$ gives

$$
\iint_{S} K\left[C^{\prime} K+4(a+c) G^{\prime}\right] d A=0 .
$$

Subtracting the last of (4.9) from (5.5), we obtain on using (2.12) and (3.6)

$$
\iint_{S}\left[K^{2} \delta+2 H\left(2 G^{\prime}-G-\bar{G}\right)\right] d A=0 .
$$

Defining $\Delta$ by the formula

$$
\Delta=\left|\begin{array}{cc}
A-A^{\prime}-\left(\bar{A}-\bar{A}^{\prime}\right) & B-B^{\prime}-\left(\bar{B}-\bar{B}^{\prime}\right) \\
B-\bar{B} & A^{\prime}-\bar{A}^{\prime}
\end{array}\right|,
$$

we find that

$$
\Delta=2 G^{\prime}-G-\bar{G} .
$$

Hence (5.6) assumes the form

$$
\iint_{S}\left(K^{2} \delta+2 H \Delta\right) d A=0 .
$$

Since $\bar{a} \bar{c}-b^{2}=a c-b^{2}$, it is known [1] that $\delta \leqq 0$ over $D$, the equality holding if and only if $a=\bar{a}, b=\bar{b}, c=\bar{c}$. Moreover use of (5.2) enables us to write (5.7) in the form

$$
\Delta=-2\left|\begin{array}{cc}
A^{\prime}-\bar{A}^{\prime} & \bar{B}-B \\
B-\bar{B} & A^{\prime}-\bar{A}^{\prime}
\end{array}\right| .
$$

It follows that $\Delta \leqq 0$. From the fact that $\delta \leqq 0$ over $D$, and from (5.8) it follows that

$$
\iint_{S} H \Delta d A \geqq 0,
$$

and from $\Delta \leqq 0$, that

$$
\iint_{S} H \Delta d A \leqq 0
$$


Hence

$$
\iint_{S} H \Delta d A=0 ; \text { therefore } \iint_{S} K^{2} \delta d A=0 .
$$

Hence $\delta=0$, and the first fundamental forms of $S$ and $\bar{S}$ are the same. Hence the homeomorphism $h$ is an isometry and therefore a rigid motion, as was to be proved.

Suppose that the Gaussian curvature $K$ is a constant. From (2.20) and (2.18) it follows that

$$
A+A^{\prime}=0, \quad B+B^{\prime}=0 .
$$

We may write (3.5) in the form

$$
G=2\left(A^{2}+B^{2}\right) \geqq 0 .
$$

Since $p \geqq 0, G \geqq 0$ it follows from the last of (4.9) that

$$
G=0, \quad p=0 \text {. }
$$

Hence $S$ is a sphere. This furnishes the promised simple proof of Liebmann's theorem.

We observe that if the associated curvature of $S$ is equal to its mean curvature then $G=0$, and $S$ is a sphere.

\section{REFERENCES}

1. S. S. Chern, Topics in differential geometry (mimeographed notes), Institute for Advanced Study, 1951.

2. - Introduction to differential geometry (mimeographed notes), the University of Chicago.

3. - Some new characterizations of the euclidian sphere, Duke Math. J. vol. 12 (1945) pp. 279-290.

4. V. G. Grove, On congruences and conjugate nets, Amer. J. Math. vol. 69 (1947) pp. 59-69.

5. S. Nakajima, Über characterische Eigenschaften der Kugel, Tôhoku Math. J. vol. 26 (1926) pp. 361-364.

Michigan State University 